\title{
Determination of partial photoionisation cross sections of methyl bromide in the first autoionisation range by use of spin polarisation photoelectron spectroscopy
}

\author{
F Schäfers $\dagger$, M A Baig $\ddagger$ and U Heinzmann $\dagger$ \\ + Fritz-Haber-Institut der Max-Planck-Gesellschaft, Faradayweg 4-6, D-1000 Berlin 33, \\ West Germany \\ $\$$ Physikalisches Institut der Universität Bonn, Nussallee 12, D-5300 Bonn 1, West \\ Germany
}

Received 12 October 1982

\begin{abstract}
Using recent experimental data on photoelectron spin polarisation combined with the high-resolution spectrum of methyl bromide, which has also been recently reported, the partial photoionisation cross sections for the ionisation of $\mathrm{CH}_{3} \mathrm{Br}$ from the $4 \pi$ ground-state orbital into the energy degenerate continua $\varepsilon \sigma, \varepsilon \pi$ and $\varepsilon \delta$ have been determined separately. Quantitative results, obtained for the autoionising resonances between 116 and $118 \mathrm{~nm}$, are reported and discussed.
\end{abstract}

Experimental work in recent years has shown that spin polarisation of photoelectrons is a rule rather than an exception for most atomic and molecular systems (for a review see Heinzmann 1980a). One way to obtain polarised photoelectrons is the Fano effect, a spin polarisation transfer from the ionising light (circularly polarised radiation) to the photoelectrons, produced even if they are extracted by an electric field regardless of their direction of emission. These studies were hampered by the fact that most atoms and molecules have their ionisation threshold in the vUV where conventional methods for producing circularly polarised radiation break down. Nevertheless, it was possible to perform such experiments using synchrotron radiation from the $2.5 \mathrm{GeV}$ Bonn electron accelerator where the light emitted above and below the synchrotron plane has been found to be largely circularly polarised (Heinzmann et al 1982).

In molecular photoionisation, such measurements have been performed with $\mathrm{CO}_{2}$ and $\mathrm{N}_{2} \mathrm{O}$ (Heinzmann et al 1980) and recently with $\mathrm{CH}_{3} \mathrm{Br}$ (Heinzmann et al 1981). Very new spin polarisation results of photoelectrons emitted from iodine and bromine molecules by unpolarised vUV radiation have just been reported (Schönhense et al 1982). The first spin polarisation calculations were performed for $\mathrm{CO}_{2}$ by Hess (Heinzmann et al 1980). For diatomic molecules, a general theoretical treatment of photoelectron spin orientation was carried out by Cherepkov (1981a, b). In these theoretical papers it was verified that the existence of a spin polarisation as well as the spin density matrix formalism expressing the spin polarisation as a function of dipole matrix elements and the phaseshift differences are both quite similar to the case of atomic photoionisation. 
In atomic photoionisation spin polarisation data and the results of photoelectron intensity spectroscopy complement each other. One thus obtains a complete set of quantum mechanical data about the photoionisation process studied, i.e. bound-free dipole matrix elements and phaseshift differences of the continuum wavefunctions (Heinzmann 1980b). Recently a high-resolution absorption spectrum of $\mathrm{CH}_{3} \mathrm{Br}$ was reported in the wavelength range $1100-1250 \AA$ (Baig et al 1982). Thus the partial photoionisation cross sections (squares of dipole matrix elements) could be determined separately for the first time for molecular photoionisation by combining this intensity spectrum with the spin polarisation data. It is the purpose of this letter to report this for $\mathrm{CH}_{3} \mathrm{Br}$; since the phaseshift difference of the continuum wavefunctions were also obtained recently (Heinzmann et al 1981), this is a further important step towards a 'complete' molecular photoionisation experiment.

The high-resolution spectrum of methyl bromide in the wavelength range 110 to $125 \mathrm{~nm}$ has been studied (Baig et al 1982) by using the $0.5 \mathrm{GeV}$ Bonn synchrotron radiation as a background source. A description of the apparatus and the experimental techniques employed in obtaining the spectra is given elsewhere (Connerade et al 1980, Baig et al 1981). In brief, they were recorded in the first order of a $3 \mathrm{~m}$ normal incidence vacuum spectrograph equipped with a 5000 lines $/ \mathrm{mm}$ holographic grating yielding a resolution of $\pm 0.0005 \mathrm{~nm}$. The main advantage of this spectroscopic technique is that the optical resolution is two orders of magnitude higher than the radiation bandwidth obtainable in spin polarisation photoelectron spectroscopy. Therefore, this is the best method of identification for absorption resonances. The disadvantage is the inaccurate determination of an absorption background lying under a resonant absorption structure or, in other words, the difficulty of finding the absolute position of the zero line in the densitometer trace of a spectrograph plate. Furthermore, absorption spectroscopy is more suited to the determination of Rydberg series limits than to that of continuum thresholds because, in molecular photoionisation, these can be different from each other in contrast to the atomic case.

On the other hand the spin polarisation technique has other advantages and disadvantages which really complement those of optical absorption spectroscopy. Experimental studies of the polarisation of photoelectrons produced by circularly polarised synchrotron radiation in Bonn used a $10 \mathrm{~m}$ normal incidence monochromator for radiation emitted out of the plane of the synchrotron. Up to $10^{10}$ photons/s have been obtained with a bandwidth of $0.08 \mathrm{~nm}$ and a degree of circular polarisation of about $80 \%$ (Heinzmann et al 1982). The photoelectrons produced at the molecular beam were extracted by an electric field regardless of their direction of emission and accelerated to $120 \mathrm{keV}$ for spin polarisation analysis in a Mott detector. The details of the experimental arrangement are described elsewhere (Heinzmann 1980b, Schäfers et al 1982). The main disadvantage of any spin polarisation analysis is that one looses an intensity factor of about $10^{3}$ in the spin detector. Therefore, all spin polarisation values have uncertainties of a few per cent due to statistical errors in the electron counting technique. An advantage of spin polarisation measurements is that, by definition, they give absolute data. Furthermore, the disadvantage that one does not get a very high wavelength resolution for resonance structures in practice, is partly offset by the fact that structures in spin polarisation resonances are always much broader than in the corresponding cross section resonances as discussed in the literature (Heinzmann and Kessler 1978, Cherepkov 1980).

The technique of spin polarisation spectroscopy within autoionising resonances is a tool to identify the nature of the resonant autoionising states and to determine their 
angular momentum quantum numbers. This has been demonstrated for the first time in the atomic photoionisation in Tl (Heinzmann et al 1975, 1976) and is illustrated here for $\mathrm{CH}_{3} \mathrm{Br}$.

The photoionisation process studied is due to the excitation of the $4 \pi$ lone-pair electron localised on a bromine site to the continuum

$$
(\mathrm{a} \sigma)^{2}(\mathrm{e} \pi)^{41} \mathrm{~A}_{1} \rightarrow(\mathrm{e} \pi)^{3}\left({ }^{2} \mathrm{E}_{3 / 2}\right) \varepsilon \lambda
$$

with

$$
\lambda=0,1,2 \quad(\sigma, \pi, \delta)
$$

or via autoionisation

$$
(\mathrm{a} \sigma)^{2}(\mathrm{e} \pi)^{4}{ }^{1} \mathrm{~A}_{1} \rightarrow(\mathrm{e} \pi)^{3}\left({ }^{2} \mathrm{E}_{1 / 2}\right) n \lambda
$$

into the continuum. Between the first two ionisation thresholds ${ }^{2} \mathrm{E}_{3 / 2}(0,0)$ and ${ }^{2} \mathrm{E}_{1 / 2}$ $(0,0)$, there is a pronounced autoionisation resonance region as known from the atomic photoionisation (see, for example, Heinzmann 1980b). In $\mathrm{CH}_{3} \mathrm{Br}$ Baig et al (1982) have recently resolved these autoionisation resonances and performed an MQDT analysis.

We have convoluted this high-resolution spectrum with a monochromator transmission profile corresponding to the $0.08 \mathrm{~nm}$ bandwidth used in the spin polarisation spectroscopy work. The resulting shape of the resonance profiles in connection with the absolute cross section of Person and Nicole (1971) enables one to determine the absolute total photoionisation cross section in the autoionisation range shown in figure 1 as the uppermost curve. It is worth noting that the shape of the pronounced

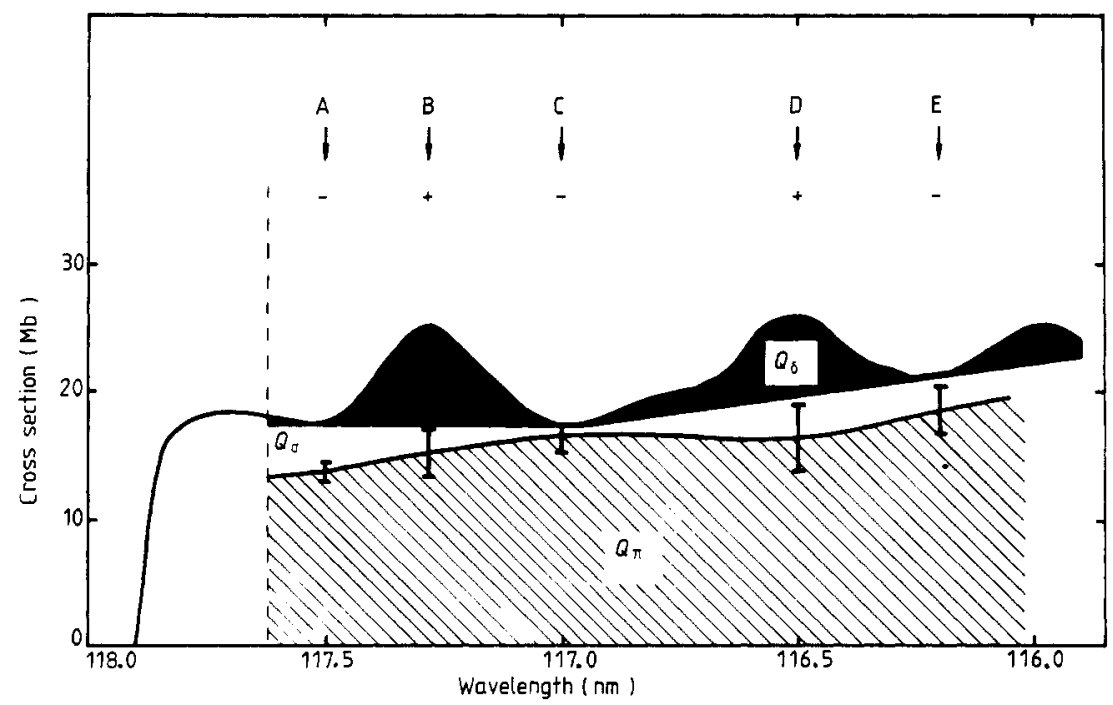

Figure 1. Partial photoionisation cross sections $Q_{\pi}$ (dashed), $Q_{\sigma}$ (white) and $Q_{\delta}$ (black) as functions of the wavelength in the first autoionisation range of $\mathrm{CH}_{3} \mathrm{Br}$. The vertical broken line indicates the position of the first photoionisation threshold (Baig et al 1982). The signs - and + show the signs of the spin polarisation values measured for photoelectrons emitted at the wavelengths $A-E$ where the total cross section has its minima and maxima. The error bars due to the uncertainty in spin polarisation data indicate the uncertainty for $Q_{\sigma}$ and $Q_{\pi}$. 
resonance structure with minima and maxima indicated by the positions $A-E$ in figure 1 is in quantitative agreement with the corresponding photoelectron intensity structures (Heinzmann et al 1981). One first consequence is, however, that the wavelengths corresponding to the photoelectron values have to be shifted by about $0.1 \mathrm{~nm}$ to longer wavelengths due to the comparison with the new highly resolved resonance data of Baig et al (1982). It is not surprising that figure 1 does not show the microstructure within the resonance profiles shown originally because of the convolution procedure mentioned above.

The signs of the spin polarisation results measured for photoionisation of $\mathrm{CH}_{3} \mathrm{Br}$ using right-handed circularly polarised radiation at the minima and the maxima (positions $\mathrm{A}-\mathrm{E}$ ) are also shown in figure 1. It is typical that all minima are connected with photoelectrons of negative spin polarisation where photon spin and electron spin are antiparallel whereas in the maxima it is the opposite. This can be explained by the corresponding polarisation formula (Heinzmann et al 1980)

$$
P=\frac{1}{2} \frac{P_{\sigma} Q_{\sigma}+P_{\pi} Q_{\pi}+P_{\delta} Q_{\delta}}{Q_{\sigma}+Q_{\pi}+Q_{\delta}}
$$

where $P_{\sigma}, P_{\pi}$ and $P_{\delta}$ are the spin polarisation values of the electrons in the partial continua $\varepsilon \sigma, \varepsilon \pi$ and $\varepsilon \delta$, respectively, and $Q_{\sigma}, Q_{\pi}$ and $Q_{\delta}$ are the corresponding partial cross sections for the transitions into the energy degenerate partial continuum orbitals. Equation (1) describes the polarisations of three final states superimposed incoherently (they are orthogonal to each other) after weighting them with the partial cross sections. The factor $\frac{1}{2}$ comes from the fact that the photoionisation process is studied at randomly oriented molecules due to integration of $P(\Theta)$ over all Euler angles (Heinzmann et al 1980).

On the assumption that the influence of the spin-orbit interaction on the continuum wavefunctions is negligible, which is a good approximation for $\mathrm{CH}_{3} \mathrm{Br}, \mathrm{CO}_{2}$ or $\mathrm{N}_{2} \mathrm{O}$ but not for molecules with heavier atoms such as $\mathrm{CH}_{3} \mathrm{I}$ (Dagata et al 1981) or $\mathrm{I}_{2}$ (Schönhense et al 1982) one obtains (Heinzmann et al 1980):

$$
P_{\sigma}=-1 \quad P_{\pi}=0 \quad P_{\delta}=+1 .
$$

From equations (1) and (2) one has

$$
P=\frac{1}{2} \frac{Q_{\delta}-Q_{\sigma}}{Q_{\delta}+Q_{\sigma}+Q_{\pi}} .
$$

In a conventional photoionisation or absorption experiment one measures the total cross section $Q=Q_{\sigma}+Q_{\pi}+Q_{\delta}$ since one cannot distinguish between transitions into different continua. A measurement of the polarisation yields, however, information on the individual channels, as one can immediately see from equation (3). If, for example, with $Q_{\pi}=0, Q_{\sigma}$ or $Q_{\delta}$ dominates, the polarisation $P$ tends to -0.5 or +0.5 , respectively. It is worth noting that $Q_{\pi}$ brings only a depolarisation effect in the denominator of equation (3) because of $P_{\pi}=0$. The positive values of the spin polarisation at the resonance maxima $\mathrm{B}$ and $\mathrm{D}$ and the negative polarisation values at the minima $\mathrm{A}, \mathrm{C}$ and $\mathrm{E}$, where destructive interference between autoionising and continuum states takes place, show that the autoionisation resonances have $\delta$ character.

Quantitatively, using the absolute new cross section results (figure 1) and the spin polarisation data one obtains the partial photoionisation cross sections $Q_{\pi}, Q_{\sigma}$ and 
$Q_{\delta}$ separately at the five positions A-E. This is shown in table 1 and figure 1 , including errors bars due to the uncertainty of the polarisation values. It has been assumed that $Q_{\sigma}$ and $Q_{\pi}$ do not show a pronounced structure opposite to $Q_{\delta}$ between the five positions A-E, if the resolution is not much better than the $0.08 \mathrm{~nm}$ used. Therefore figure 1 shows dashed and white areas for $Q_{\pi}$ and $Q_{\sigma}$, respectively, additional to the black area for the $Q_{\delta}$ partial cross section.

Table 1. Experimental values of the spin polarisation of photoelectrons and of the partial and total photoionisation cross sections for $\mathrm{CH}_{3} \mathrm{Br}$ at the wavelengths $\mathrm{A}-\mathrm{E}$ where the total cross section has its minima and maxima.

\begin{tabular}{lllllllll}
\hline Position & $\begin{array}{l}\text { Wavelength } \\
(\mathrm{nm})\end{array}$ & $\begin{array}{l}\text { Polarisation } \\
(\%)\end{array}$ & $\begin{array}{l}Q_{\sigma} \\
(\mathrm{Mb})\end{array}$ & $\begin{array}{l}Q_{\pi} \\
(\mathrm{Mb})\end{array}$ & $\begin{array}{l}Q_{\sigma}+Q_{\pi} \\
(\mathrm{Mb})\end{array}$ & $\begin{array}{l}Q_{\delta} \\
(\mathrm{Mb})\end{array}$ & $\begin{array}{l}Q_{\delta}^{0} \\
(\mathrm{Mb})\end{array}$ & $\begin{array}{l}Q_{\sigma}+Q_{\pi}+Q_{\delta} \\
(\mathrm{Mb})\end{array}$ \\
\hline $\mathrm{A}$ & 117.5 & $-10 \pm 2$ & $3.4 \pm 0.8$ & $13.6 \neq 0.8$ & 17.0 & 0 & & 17.0 \\
$\mathrm{~B}$ & 117.3 & $+12 \pm 5$ & $2.0 \pm 2.0$ & $15.3 \neq 2.0$ & 17.3 & 8.0 & 0.2 & 25.3 \\
$\mathrm{C}$ & 117.0 & $-2 \pm 5$ & $0.7 \pm 1.8$ & $17.0 \neq 1.8$ & 17.7 & 0 & & 17.7 \\
$\mathrm{D}$ & 116.5 & $+5 \pm 5$ & $3.5 \pm 2.5$ & $16.3 \neq 2.5$ & 19.8 & 6.1 & 0.16 & 25.9 \\
$\mathrm{E}$ & 116.2 & $-6 \pm 5$ & $2.5 \pm 2.0$ & $18.6 \neq 2.0$ & 21.1 & 0 & 21.1 \\
\hline
\end{tabular}

Assuming the first two autoionisation resonances to be isolated the shape of the resonance structure measured by Baig et al (1982) can be fitted as a Fano-type resonance with the following resonance parameters: resonance wavelengths 117.31 and $116.58 \mathrm{~nm}$, resonance widths $\Gamma=0.25$ and $0.21 \mathrm{~nm}$ and $q$ values 6.2 and 6.0 , respectively. In the maxima of the resonance cross section one obtains

$$
Q_{\delta}=Q_{\delta}^{0}\left(1+q^{2}\right)
$$

where $Q_{\delta}^{o}$ is the undisturbed partial photoionisation cross section if there is no coupling between continuum and autoionising states. Using equation (4), the $q$ values obtained and the results of $Q_{\delta}$ given in table 1 one obtains

$$
Q_{\delta}^{0}=0.2 \mathrm{Mb} \quad \text { for position } \mathrm{B}
$$

and

$$
Q_{\delta}^{0}=0.16 \mathrm{Mb} \quad \text { for position } \mathrm{D} \text {. }
$$

These values are also listed in table 1 in a separate column.

One surprising result shown in figure 1 is that $Q_{\pi}$ is larger than $Q_{\sigma}$. For comparison a single-excitation $\mathrm{CI}$ calculation starting from self-consistent-field wavefunctions for $\mathrm{CO}_{2}$ (Buenker and Peyerimhoff 1974) yields $1.5,1$ and $0.1 \mathrm{Mb}$ respectively as partial cross sections for ionisation to $\sigma, \pi$ or $\delta$ continuum states at the Koopman's theorem ionisation potential of $14.7 \mathrm{eV}$ (Heinzmann et al 1980). Ab initio calculations, especially for $\mathrm{CH}_{3} \mathrm{Br}$, would be necessary to describe these relations between $Q_{\sigma}, Q_{\pi}$ and $Q_{\delta}$ or between the corresponding dipole matrix elements.

There is another open question from the experimental point of view. Baig et al (1982) have found a series limit in photoabsorption at $117.623 \mathrm{~nm}$, shown in figure 1 as a vertical broken line, and have also interpreted it as a photoionisation threshold. On the other hand there is no doubt that photoelectrons have also been obtained between 117.6 and $117.9 \mathrm{~nm}$ indicated by the cross section curve in figure 1 beyond the series limit shown. This may be due to an excited molecular state producing so called 'hot' electrons. 
To resolve this discrepancy and decide what type of state is involved at the photoionisation threshold of $\mathrm{CH}_{3} \mathrm{Br}$ between 117.6 and $117.9 \mathrm{~nm}$, the new technique of spin polarisation threshold electron spectroscopy (Heckenkamp et al 1983) could probably be applied. Further work with the new German dedicated storage ring for synchrotron radiation, BESSY, where two orders of magnitude more intensity of circularly polarised synchrotron radiation are available will enable us to continue spin-resolved photoelectron spectroscopy which will also be emission angle resolved. The results of this letter show that halogen compounds are very interesting examples in the search for a complete quantum mechanical parameter set to describe the molecular photoionisation process studied.

We express our gratitude to the Bundesminister für Forschung und Technologie BMFT for financial support.

\section{References}

Baig M A, Connerade J P, Dagata J A and McGlynn S P 1981 J. Phys. B: At. Mol. Phys. 14 L25

Baig M A, Connerade J P and Hormes J 1982 J. Phys. B: At. Mol. Phys. 15 L5-10

Buenker R J and Peyerimhoff S D 1974 Theor. Chim. Acta 3533

Cherepkov N A 1980 Opt. Spectrosc. 49 1067-75

- 1981a J. Phys. B: At. Mol. Phys. 14 L73-7

- 1981b J. Phys. B: At. Mol. Phys. 14 2165-77

Connerade J P, Baig M A, Garton W R S and McGlynn S P 1980 J. Phys. B: At. Mol. Phys. 13 L705

Dagata J A, Findley G L, McGlynn S P, Connerade J P and Baig M A 1981 Phys. Rev. A 24 2485-90

Heckenkamp Ch, Schäfers F, Heinzmann U, Frey R and Schlag E W 1983 Nucl. Instrum. Meth. to be published

Heinzmann U 1980a Appl. Opt. 19 4087-91

1980b J. Phys. B: At. Mol. Phys. 13 4353-85

Heinzmann U, Heuer H and Kessler J 1975 Phys. Rev. Lett. 34 441-4, 710

— 1976 Phys. Rev. Lett. 36 1444-7

Heinzmann U and Kessler J 1978 J. Phys. B: At. Mol. Phys. 11 L265-6

Heinzmann U, Osterheld B and Schäfers F 1982 Nucl. Instrum. Meth. 195 395-8

Heinzmann U, Osterheld B, Schäfers F and Schönhense G 1981 J. Phys. B: At. Mol. Phys. 14 L79-84

Heinzmann U, Schäfers F and Hess B A 1980 Chem. Phys. Lett. 69 284-9

Person J C and Nicole P P 1971 J. Chem. Phys. 55 3390-7

Schäfers F, Schönhense G and Heinzmann U 1982 Z. Phys. A 304 41-8

Schönhense G, Heckenkamp Ch and Heinzmann U 1982 Proc. 8th Int. Conf. on Atomic Physics, Göteborg ed I Lindgren et al (Lund: Wallin and Dalholm Boktr, AB) Abstracts B16 